\title{
Análisis de los riesgos asociados al uso de la técnica VMAT frente a 3DCRT en tratamientos de mama con cadenas ganglionares axilo-claviculares
}

\section{Risk analysis associated with the use of the VMAT technique vs. 3DCRT in breast treatments with axillary and supraclavicular lymph nodes}

\author{
Paz García de Acilu Laá1, Juan García Ruíz-Zorrilla', Ángel Montero Luis², Raquel Ciérvide Jurio², \\ Carmen Rubio Rodríguez ${ }^{1,2}$, Pedro Fernández Letón ${ }^{1,2}$ \\ 1 Hospital Universitario HM Puerta del Sur, Móstoles (Madrid) \\ 2 Hospital Universitario HM Sanchinarro, Madrid
}

Fecha de Recepción: 15/04/2020 - Fecha de Aceptación: 23/11/2020

La utilización de arcoterapia volumétrica de intensidad modulada (VMAT) para el tratamiento de cáncer de mama siempre ha sido un asunto controvertido. La mayor conformación de las dosis absorbidas altas que se consigue con la VMAT frente a la radioterapia conformada (3DCRT) viene acompañada de un incremento del volumen irradiado con dosis absorbidas bajas, cuyas consecuencias hay que analizar. El presente estudio pretende, mediante el análisis de las dosimetrías clínicas de 80 pacientes con cáncer de mama tratadas en nuestro centro con ambas técnicas, evaluar la idoneidad de la elección de una técnica frente a la otra para el tratamiento de pacientes con cáncer de mama que precisan la irradiación de cadenas ganglionares axilo-claviculares, pero no de la cadena mamaria interna. Para ello se tienen en cuenta por un lado criterios puramente dosimétricos, como el cumplimento de los límites de dosis absorbida establecidos en órganos de riesgo, y por otro se realiza una estimación del aumento en la probabilidad de desarrollar tumores secundarios sólidos por la irradiación a baja dosis de órganos sanos y se evalúan las posibles la toxicidades, cardiacas y pulmonares, en base a lo descrito en la literatura.

Palabras clave: VMAT, 3DCRT, cáncer de mama, tumores radioinducidos, cardiotoxicidad.

The use Volumetric Intensity Modulated ArcTherapy (VMAT) for breast cancer treatments has always been controversial. The tighter conformation of the high absorbed doses around the target that VMAT provides over conformed radiotherapy (3DCRT) entails an increase of the healthy volumes irradiated with low doses, and the consequences of this fact should be analysed. This study tries to assess the suitability of one or another technique for the treatment of breast cancer patients with PTV volumes involving axillary and supraclavicular lymph nodes but not the internal mammary chain. For this purpose, treatment plans of 80 patients that have been treated at our Institution with both techniques were analysed. First of all, pure dosimetric criteria were taken into account, for instance keep the dose in the organs at risk under the established limits. On the other hand, an estimation of the increase in the probability of developing secondary solid radio-induced tumours, due to low dose irradiation of healthy tissues, was done. Heart and pulmonary toxicities were also discussed according to the state of the art.

Key words: VMAT, 3DCRT, breast cancer, radio-induced tumours, cardiotoxicity.

\section{Introducción}

El uso de técnicas de intensidad modulada (intensity modulated radiotherapy, IMRT) en tratamientos de radioterapia ha ido en aumento en los últimos 10 años, llegando a constituir el tratamiento estándar para algunas patologías. En nuestro centro se ha pasado de tratar con IMRT a un 30\% de nuestros pacientes en el

\footnotetext{
*Correspondencia: pgarciadeacilu@hmhospitales.com https://doi.org/10.37004/sefm/2021.22.1.001
} 
año 2008 a un 60\% en 2019. Sin embargo, en algunas localizaciones, como es el caso de la irradiación de mama con cadenas ganglionares axilo supraclaviculares ipsilaterales (niveles ganglionares I-IV) sin inclusión de la cadena ganglionar mamaria interna, no existe un consenso entre la comunidad científica sobre la idoneidad de este tipo de tratamientos frente a la radioterapia conformada tridimensional (3D Dimensional Conformal Radiotherapy, 3DCRT). ${ }^{1-5}$

La IMRT, mediante la diversificación de las entradas de los haces, ya sea con múltiples incidencias o mediante arcos en IMRT volumétrica (Volumetric Intensity ModulatedArcTherapy, VMAT), permite conformar las dosis medias y altas (valores entre $25-85 \%$ y superiores al $85 \%$ de la de prescripción respectivamente) en las inmediaciones de los volúmenes objetivo, reduciéndose dicha dosis en los órganos circundantes y limitando así la aparición de gran parte de los efectos deterministas que causa la radiación sobre los tejidos sanos. Concretamente en la irradiación de mama las toxicidades más comunes se producen por la irradiación con dosis altas de la piel (eritemas, descamación de la piel, hiperpigmentación y telangiectasia) $)^{6,7}$ y con dosis medias-altas de los pulmones ${ }^{8,9}$ (neumonitis 0 fibrosis pulmonar).

Por otra parte, el uso de radioterapia de intensidad modulada, especialmente VMAT, presenta también una desventaja importante frente a la 3DCRT, ya que genera una distribución de dosis absorbidas bajas en zonas que no se desea tratar. Este baño de dosis absorbidas bajas ( $\leq 10 \mathrm{~Gy}$ ) a las que se somete a la paciente aumenta la probabilidad de sufrir efectos estocásticos, cuya incidencia no tiene una dosis absorbida umbral pero cuya probabilidad se incrementa con la dosis integral recibida. Además pese a reducirse el volumen de órganos sanos que recibe una dosis alta, la dosis absorbida promedio que reciben determinados órganos, como pulmones y corazón, puede ser superior. ${ }^{2,10}$

En el caso concreto de las pacientes con cáncer de mama, la supervivencia a largo plazo es elevada. En Europa norte, central y sur la supervivencia neta a 5 años se encuentra entre el 82 y el $85 \%$, mientras que es algo inferior en USA (84\%) y en Europa del este (72\%). En casos de enfermedad localizada esta cifra asciende hasta el 99\%. . $^{11,12}$ Estos datos de supervivencia, unidos al hecho de que en mama las toxicidades agudas asociadas a la radioterapia, excepto en la piel, son infrecuentes, ${ }^{13,14}$ hacen que a la hora de escoger la técnica de tratamiento para cada paciente sea necesario valorar las consecuencias a largo plazo de las dosis absorbida bajas, y la posible aparición de toxicidades crónicas. ${ }^{15}$

El objetivo del presente trabajo es evaluar las consecuencias que puede acarrear la distinta forma que tiene de distribuirse la dosis sobre los órganos de riesgo con la planificación 3DCRT en comparación con la planificación mediante VMAT. Para ello se comparan, en primer lugar, los criterios puramente dosimétricos de planificaciones clínicas de pacientes con cáncer de mama realizadas en nuestros centros con ambas técnicas. En segundo lugar se realiza una estimación del exceso de riesgo que conlleva, en cuanto a la formación de tumores radioinducidos y episodios cardiovasculares severos se refiere, el incremento del volumen irradiado con dosis bajas que implica el uso de VMAT.

\section{Material y método}

Se han analizado de forma consecutiva un total de 80 dosimetrías clínicas de pacientes con cáncer de mama tratadas durante los años 2018 y 2019, siendo 40 de ellas tratamientos de mama izquierda y las 40 restantes tratamientos de mama derecha. Las dosimetrías clínicas se realizaron con el sistema de planificación de radioterapia Raystation de RaySearch (RaySearch Laboratories, Estocolmo, Suecia). La mitad de las planificaciones evaluadas se realizaron con 3DCRT y la otra mitad con VMAT, y todos los haces y arcos utilizados tienen energía de 6 MV.

El procedimiento seguido en nuestro centro a la hora de abordar el tratamiento de las pacientes subsidiarias de recibir radioterapia sobre la mama y las cadenas ganglionares sin inclusión de la cadena de la mamaria interna, es iniciar por defecto la dosimetría clínica mediante técnica de 3DCRT. Tras realizar una primera aproximación del tratamiento, estableciendo la posición del isocentro y de los dos haces tangenciales, se evalúa si es posible cumplir los límites de dosis absorbida establecidos para los órganos de riesgo (OAR), tabla 1. Los valores mostrados en dicha tabla fueron establecidos por consenso en nuestro servicio basándose en el documento RTOG-1005, en las recomendaciones del Grupo Español de Oncología Radioterápica de Mama (GEORM) y de la Sociedad

Tabla 1. Límites de Dosis absorbida en OAR (RTOG-1005 y GEORM-SESPM).

\begin{tabular}{c|c} 
Mama Contralateral & V4 $<30 \%$ \\
\hline \multirow{4}{*}{ Pulmón Ipsilateral } & $\mathrm{V} 16<25 \%$ \\
\cline { 2 - 2 } & $\mathrm{V} 8<45 \%$ \\
\cline { 2 - 2 } & $\mathrm{V} 4<60 \%$ \\
\hline $\begin{array}{c}\text { Pulmón } \\
\text { Contralateral }\end{array}$ & $\mathrm{D} 4<17 \mathrm{~Gy}$ \\
\hline \multirow{2}{*}{ Corazón } & $D_{\text {media }}<6.5 \mathrm{~Gy}$ \\
\hline Canal Medular & $\mathrm{V} 16<5 \%$ \\
\cline { 2 - 2 } & $\mathrm{V} 8<30 \%$ \\
\hline
\end{tabular}


Tabla 2. Pacientes incluidas en el estudio con y sin boost clasificadas en función de lateralidad y técnica.

\begin{tabular}{c|c|c|cc}
\multirow{2}{*}{} & \multicolumn{2}{|c|}{ Mama derecha } & \multicolumn{2}{c}{ Mama izquierda } \\
\cline { 2 - 5 } & 3D & VMAT & 3D & VMAT \\
\hline Con B00ST & 12 & 9 & 11 & 10 \\
\hline Sin B00ST & 8 & 11 & 9 & 10
\end{tabular}

Española de Senología y Patología Mamaria (SESPM) y en nuestra propia experiencia. En caso de no ser posible mantenerse dentro de estos límites, generalmente por incumplimiento del corazón y/o del pulmón ipsilateral, la dosimetría se realiza mediante técnica de VMAT.

Las pacientes planificadas con técnica VMAT se trataron en los aceleradores Versa e Infinity de Elekta, ambos equipados con el multiláminas Agility (Elekta Instrument $\mathrm{AB}$ Estocolmo, Suecia), mientras que las pacientes planificadas con 3DCRT se trataron o bien en los aceleradores citados previamente o bien en el acelerador Oncor de Siemens (Siemens Healthcare $\mathrm{GmbH}$, Erlangen, Alemania).

En cuanto a los volúmenes de tratamiento, se incluyen la glándula mamaria o pared de mastectomía y las cadenas ganglionares axilo-supraclaviculares ipsilaterales (excepto la cadena ganglionar mamaria interna).
EI PTV de la mama se limita en su parte anterior retrayendo $3 \mathrm{~mm}$ desde el externo para excluir la piel y en la parte posterior excluyendo la pared costal.

La dosis prescrita a los PTVs es 40.50 Gy en 15 fracciones de 2.7 Gy. En los tratamientos realizados tras cirugía conservadora se realizó además una sobreimpresión o boost concomitante al que se prescribieron 48 Gy. De las 80 pacientes analizadas aproximadamente la mitad incluían esta sobreimpresión (tabla 2).

El criterio de cobertura mínimo para considerar una dosimetría aceptable es que el 95\% del volumen de cada uno de los PTVs esté cubierto por el 95\% de la dosis de prescripción (V95 > 95\%), aunque generalmente las coberturas alcanzadas se encuentran en valores del 98-99\%. Además el volumen que recibe sobredosificaciones del $107 \%$ de la dosis de prescripción debe ser inferior al 5\% del PTV (V107 < 5\%), salvo en el caso de que exista boost concomitante.

Desde el punto de vista dosimétrico, los tratamientos de 3DCRT se planificaron con un isocentro compartido situado entre la mama y la fosa supraclavicular. La mama y axila inferior se trataron con dos haces tangenciales mientras que la irradiación de la fosa y axila superior se realizó con 5 haces con incidencias ipsilaterales. En ambas partes se utilizan segmentos de menor tamaño para homogeneizar la distribución de dosis. En caso de tener boost concomitante la irradiación del mismo se realiza con un mínimo de 3 haces (fig. 1).
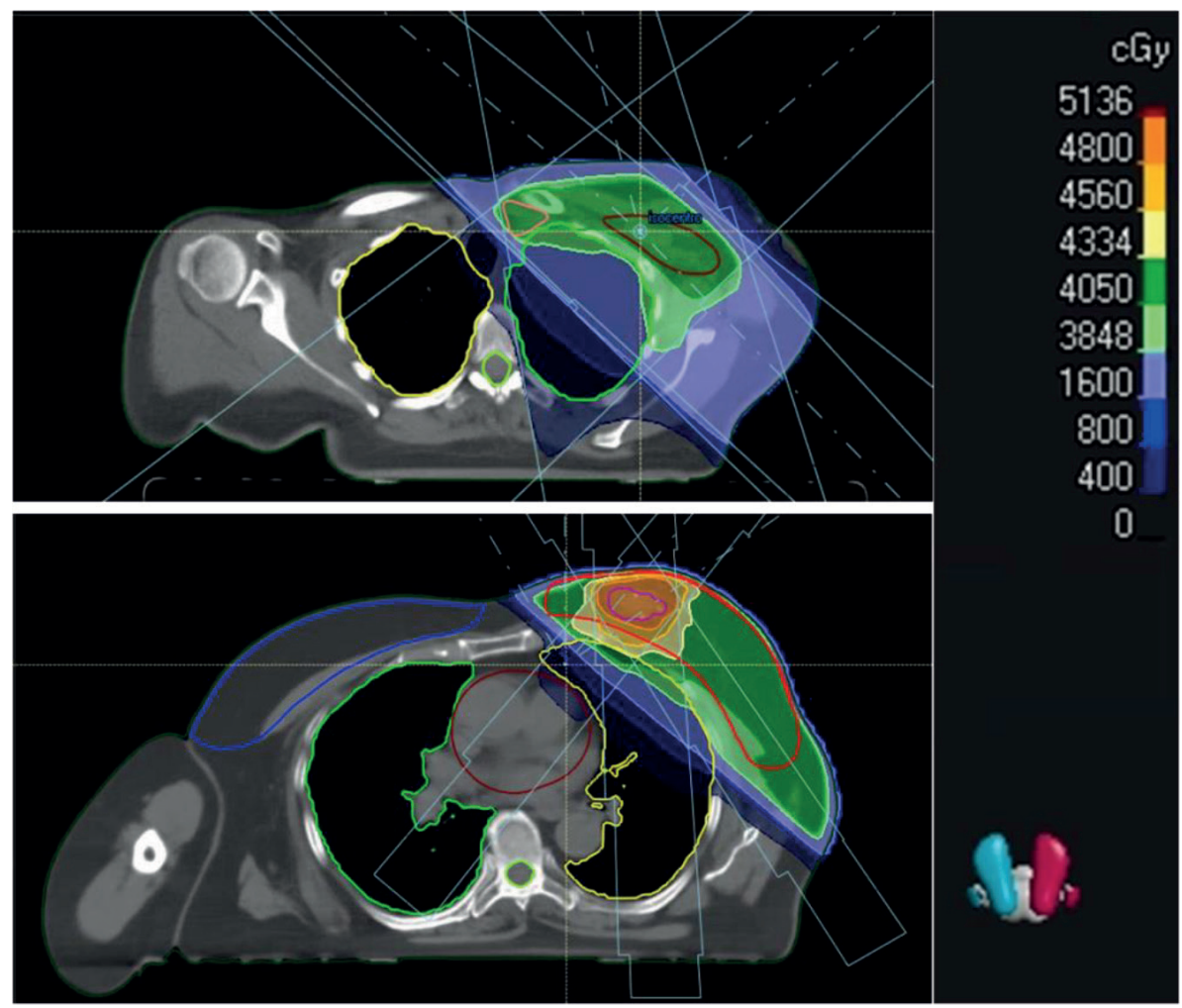

Fig. 1. Distribución de los haces y curvas de isodosis sobre el TAC de una paciente tratada con 3DCRT. 

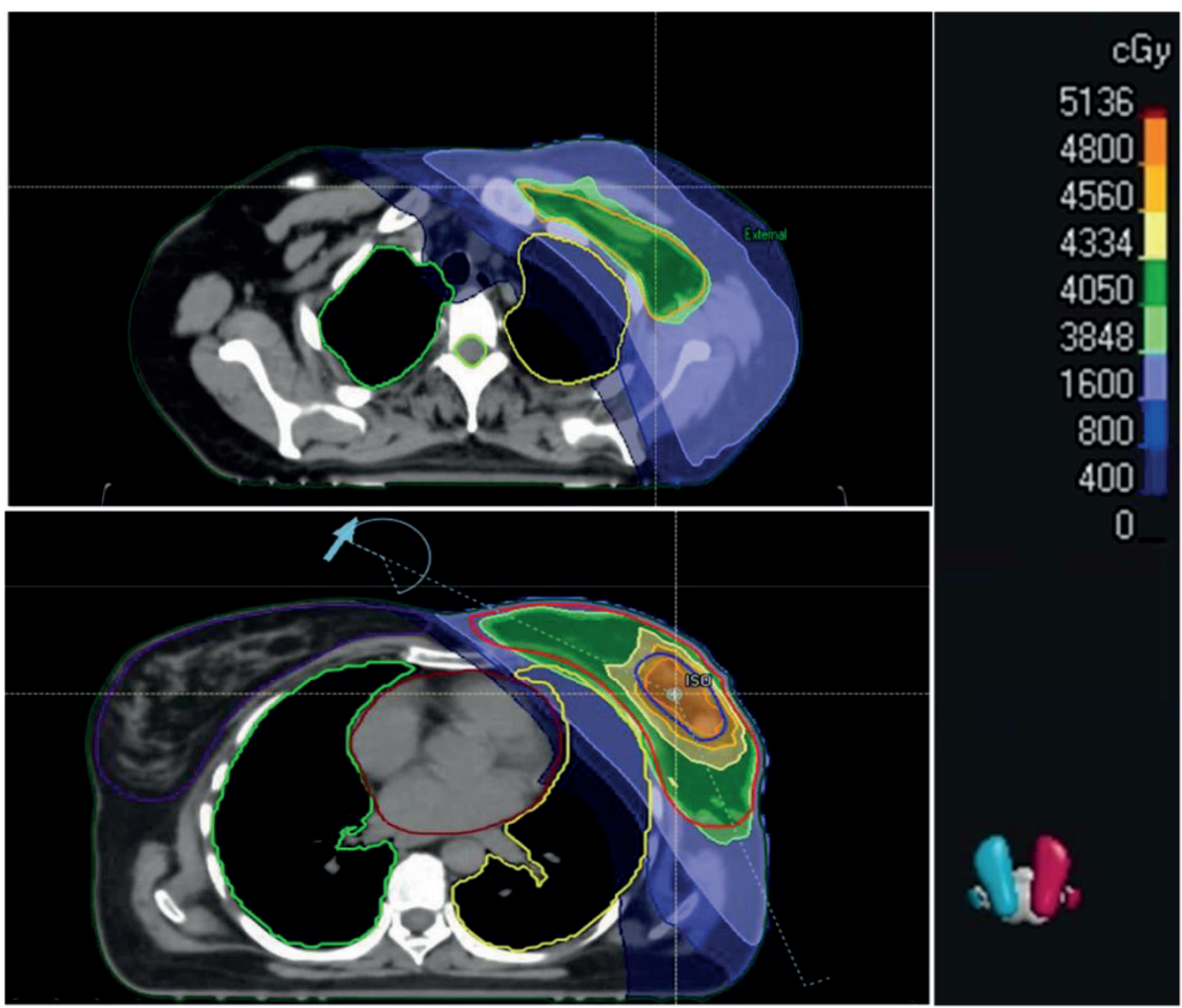

Fig. 2. Esquema de los arcos y curvas de isodosis sobre el TAC de una paciente tratada con VMAT.

Los tratamientos planificados con VMAT se realizaron con dos arcos de aproximadamente $250^{\circ}$, que abarcan desde la incidencia tangencial interna a prácticamente una incidencia posterior. El isocentro en estos casos se sitúa aproximadamente en el punto medio de los volúmenes a tratar en dirección cráneocaudal, y dentro del PTV de mama (fig. 2).

Con el objetivo de cuantificar las consecuencias que puede tener el aumento de dosis absorbidas bajas que proporciona al paciente el uso de técnicas volumétricas se siguió la metodología de Schneider et al., ${ }^{16,17}$ según la cual es posible estimar el Exceso de Riesgo Absoluto de desarrollar un segundo tumor sólido tras la radioterapia (EAR por 10000 personas y año) a partir de la dosis equivalente en órgano (OED),

$$
\mathrm{EAR}=\mathrm{EAR}_{0} \cdot \mathrm{OED}
$$

siendo $\mathrm{EAR}_{0}$, el exceso absoluto de riesgo de cáncer radioinducido para el órgano específico a dosis absorbidas bajas, por 10000 personas por Gy y año. Este parámetro incluye una dependencia con la edad actual, la edad en el momento de la exposición y el sexo de la persona irradiada.

El $\mathrm{EAR}_{0}$ se podría estimar individualmente para cada paciente, teniendo en cuenta su edad actual y la edad en el momento del tratamiento, sin embargo esa manera de proceder nos llevaría a una gran dispersión en los valores del EAR, ya que las pacientes seleccionadas no se irradiaron con edades similares. Para reducir esta variabilidad y evaluar solamente la influencia de la distribución de dosis recibida por los órganos de riesgo, consideraremos para todos los casos el mismo $\mathrm{EAR}_{0}$, que correspondería a mujeres de 70 años que recibieron el tratamiento cuando tenían 30 años. ${ }^{18}$ Para ese caso, los $\mathrm{EAR}_{0}$ estimados a partir de los datos de supervivientes a las bombas atómicas y corregidos para adecuar de las características de la población japonesa a las de la población occidental son 8.2 para la mama contralateral y 8.0 para los pulmones. ${ }^{19,20}$

La estimación de la OED puede realizarse con distinto modelos. Para dosis absorbidas inferiores a 2 Gy, se puede considerar que el número de células que mutan y dan lugar a un crecimiento tumoral es proporcional a la dosis absorbida recibida, y por tanto la relación entre tumores radioinducidos y dosis absorbida recibida sigue un comportamiento lineal. Sin embargo, para dosis absorbidas superiores a 2 Gy, la relación dosis-respuesta para carcinogénesis ya no se comporta de modo lineal, debido a la muerte celular y la esterilización de parte de las células mutadas. Además en un modelo de OED aplicado en radioterapia debe tenerse en cuenta la influencia del fraccionamiento de la dosis y la reparación y repoblación celular. 
En este trabajo la OED se ha estimado con tres modelos, linear-exponencial, plateau y un modelo mecánico más completo descritos en los trabajos de Schneider et al.20,21

El modelo linear-exponencial considera que la probabilidad de muerte celular aumenta exponencialmente con la dosis, y limita por tanto de esta manera el crecimiento lineal de la probabilidad de desarrollar tumores radioinducidos con la dosis recibida.

$$
\mathrm{OED}_{L-E}=\frac{1}{V} \sum_{i} V\left(D_{i}\right) \cdot D_{i} \cdot e^{-\alpha^{\prime} \cdot D_{i}}
$$

El modelo plateau asume que, tras el crecimiento lineal de la OED con la dosis absorbida, se alcanza una zona de plateau debido al equilibrio entre la muerte celular y los efectos de recuperación

$$
\mathrm{OED}_{\text {plateau }}=\frac{1}{V} \sum_{i} V\left(D_{i}\right) \cdot\left(1-e^{-\alpha^{\prime} \cdot D_{i}}\right) / \alpha^{\prime}
$$

El modelo mecánico incluye además la influencia del número de fracciones a la hora de tener en cuenta la repoblación y reparación celular

$$
\begin{gathered}
\mathrm{OED}_{\text {mecánico }}=\frac{1}{V} \sum_{i} V\left(D_{i}\right) \cdot \frac{e^{-\alpha^{\prime} D_{i}}}{\alpha^{\prime} R} \\
\cdot\left[1-2 R+R^{2} e^{\alpha^{\prime} D_{i}}-(1-R)^{2} e^{-\alpha^{\prime} R D_{i} / 1-R}\right]
\end{gathered}
$$

En las ecuaciones 2-4, $V\left(D_{i}\right)$ representa el volumen de órgano que recibe una dosis $D_{i}$ y $V$ es el volumen total del órgano.

El parámetro $R$ representa la repoblación celular, siendo $R=0$ la ausencia de repoblación/reparación celular entre fracciones y $R=1$ la repoblación/reparación total. Por otra parte $\alpha^{\prime}$ representa los efectos de destrucción celular y se define utilizando el modelo linear cuadrático

$$
\alpha^{\prime}=\alpha+\beta \frac{D}{N}
$$

siendo $N$ el número de fracciones y asumiendo un valor de $\alpha / \beta=3$ para todos los tejidos.

Los parámetros $\alpha$ y $R$ que se utilizar en el cálculo de los OED no son únicos. En la literatura se encuentran valores de ajuste diferentes extraídos a partir de distintos conjuntos de pacientes de Hodgkin tratados con radioterapia. ${ }^{20,22,23}$ En este estudio utilizamos los parámetros los parámetros más recientes proporcionados por Schneider (ver tabla 3). ${ }^{20}$
Tabla 3. Parámetros utilizados en el cálculo de la OED.

\begin{tabular}{c|c|c|c|c} 
& L-E & Plateau & \multicolumn{2}{|c}{ Mecánico } \\
\cline { 2 - 5 } & $\boldsymbol{\alpha}\left(\mathrm{Gy}^{-1}\right)$ & $\boldsymbol{\alpha}\left(\mathrm{Gy}^{-1}\right)$ & $\mathrm{R}$ & $\boldsymbol{\alpha}\left(\mathrm{Gy}^{-1}\right)$ \\
\hline Mama & 0.041 & 0.115 & 0.15 & 0.044 \\
\hline Pulmón & 0.022 & 0.056 & 0.83 & 0.042
\end{tabular}

En las pacientes de mama izquierda además se realizó una estimación del Exceso de Riesgo Acumulado (CER) de sufrir un episodio cardiovascular severo (ACE, Acute Coronary Event) en los 9 años posteriores a recibir el tratamiento radioterápico en función de la dosis absorbida media recibida por el corazón, entendiendo como ACE un diagnóstico de infarto de miocardio, revascularización coronaria o muerte por enfermedad isquémica.

Para ello se utilizan las expresiones presentadas por Bogaard et al. ${ }^{24}$ (ecuaciones 6-9). El modelo incluye, además de la dosis media del corazón, la influencia de la edad de la paciente en el momento del tratamiento y la existencia de factores de riesgo para ACE previos a la irradiación (historial de enfermedad isquémica, hipertensión, embolismo pulmonar, diabetes, hábito tabáquico o sobrepeso). En el citado trabajo de Boogard se validaban los resultados de Darby et al. ${ }^{25}$ respecto a la dependencia de la probabilidad de ACE con la dosis absorbida media a corazón, y por tanto seguía su metodología, considerando los factores previos de riesgo como una variable booleana: 0 si no existen factores de riesgo previos y 1 si existía uno o más de estos factores. En el mismo trabajo presentaban un modelo predictivo más avanzado, que no depende de la dosis media sino del volumen de ventrículo derecho que recibe 5 Gy, y que incluye además una ponderación de los factores de riesgo previos. Nosotros hemos utilizado el modelo inicial, dependiente de dosis media, ya que es la magnitud que tenemos evaluada en nuestras dosimetrías.

Se define un factor predictivo linear, LP, como

$$
\mathrm{LP}=0.153 \cdot D_{\text {media }}+0.087 \cdot E+1.82 \cdot R
$$

donde la $D_{\text {media }}$ se expresa en Gy, la edad $E$ en años y $R(0 \circ 1)$ representa los factores de riesgo de ACE previos al tratamiento.

La Incidencia Acumulada de ACE en los 9 años posteriores al tratamiento se calcula mediante

$$
\mathrm{CI}_{9} \text { años }=1-e^{-0.000025 \cdot e^{\mathrm{LP}}}
$$


y por tanto el Exceso de Riesgo Absoluto debido a la existencia de un tratamiento radioterápico se puede estimar con

$$
\mathrm{CEAR}_{9 \text { años }}=\mathrm{CI}_{9 \text { años }}-\mathrm{CI}_{9 \text { años, } 0 \text { Gy }}
$$

y el Exceso de Riesgo Relativo sería

$$
\mathrm{CERR}_{9} \text { años }=\mathrm{CI}_{9 \text { años }} / \mathrm{CI}_{9 \text { años, } 0 \text { Gy }}-1
$$

Para cada paciente se exportó el histograma dosis volumen a una base de datos para su almacenamiento y explotación como se describe a continuación. Los parámetros dosimétricos evaluados son: los volúmenes de cobertura (V95) y sobredosificaciones (V107) de los PTVs, en el corazón la dosis absorbida media $\left(D_{\text {media }}\right)$, dosis que recibe el $2 \%$ del volumen $\left(D_{2 \%}\right)$ y el volumen que recibe 4, 8 y 16 Gy (V4, V8, y V16) y en el pulmón ipsilateral la $D_{\text {media }}$ y $\mathrm{V} 16$. Además se calcula el EAR, considerando la contribución de la OED de la mama contralateral y de ambos pulmones y en casos de mama izquierda se calculan el $\mathrm{CERR}_{9}$ años $\mathrm{y}$ el CEAR ${ }_{9}$ años.

Se ha evaluado también la dosis absorbida media y máxima (definida como dosis que recibe el 1\% del volumen) para la arteria descendiente anterior (DA), sin embargo tanto la delimitación de la misma como la evaluación de la dosis se realizó a posteriori, no teniendo en cuenta este órgano durante la planificación.

\section{Resultados}

La cobertura y sobredosificaciones obtenidas con los dos tipos de planificación son muy similares, como se puede ver en las tablas 4-5 y la fig. 3a, y no justifica por sí sola la elección de una técnica sobre la otra.

Se encuentra que el V107 en la mama en tratamientos con boost integrado es menor con VMAT, debido a la mayor conformación de la dosis prescrita al

\begin{tabular}{|c|c|c|c|c|c|}
\hline & & & PTV MAMA & PTV AXILA & PTV FOSA \\
\hline \multirow{4}{*}{ MAMA IZQUIERDA } & \multirow{2}{*}{3 DCRT } & V95 (\%) & $97.0 \pm 1.4$ & $98.0 \pm 1.8$ & $97.7 \pm 1.3$ \\
\hline & & V107 (\%) & $0.5 \pm 0.9$ & $0.4 \pm 0.8$ & $0.1 \pm 0.2$ \\
\hline & \multirow{2}{*}{ VMAT } & V95 (\%) & $96.7 \pm 1.5$ & $98.8 \pm 1.2$ & $98.8 \pm 1.1$ \\
\hline & & V107 (\%) & $1.1 \pm 1.0$ & $0.7 \pm 1.4$ & $2 \pm 4$ \\
\hline \multirow{4}{*}{ MAMA DERECHA } & \multirow{2}{*}{3 DCRT } & V95 (\%) & $98.3 \pm 1.2$ & $98.2 \pm 0.8$ & $98.7 \pm 0.8$ \\
\hline & & V107 (\%) & $0.11 \pm 0.12$ & $0.2 \pm 0.4$ & $0.1 \pm 0.1$ \\
\hline & \multirow{2}{*}{ VMAT } & V95 (\%) & $97.0 \pm 1.4$ & $98.1 \pm 1.4$ & $98.6 \pm 1.0$ \\
\hline & & V107 (\%) & $1.3 \pm 1.5$ & $0.3 \pm 0.7$ & $0.09 \pm 0.14$ \\
\hline
\end{tabular}

Tabla 4. Porcentajes de PTVs cubiertos por el 95\% y el 107\% de las dosis de prescripción en tratamientos sin boost.
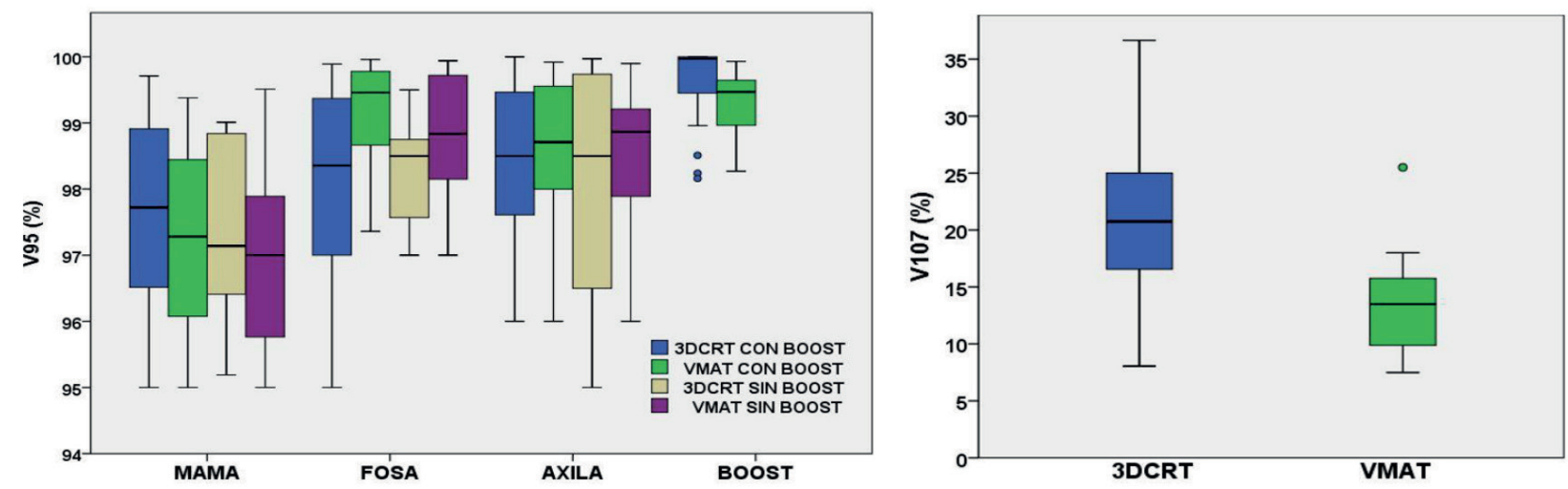

Fig. 3. a) Volúmenes de cobertura (V95) de los PTVs para casos con y sin boost, planificados con 3DCRT y VMAT. b) Volumen de sobredosificación (V107) de mama, para casos con boost, planificados con 3DCRT y VMAT. 
Tabla 5. Porcentajes de PTVs cubiertos por el 95\% y el 107\% de las dosis de prescripción en tratamientos con boost.

\begin{tabular}{|c|c|c|c|c|c|c|}
\hline & & & PTV MAMA & PTV AXILA & PTV FOSA & PTV BOOST \\
\hline \multirow{4}{*}{ MAMA IZQUIERDA } & \multirow{2}{*}{3 DCRT } & V95 (\%) & $97.8 \pm 1.4$ & $98.8 \pm 1.1$ & $98.1 \pm 1.2$ & $99.6 \pm 0.6$ \\
\hline & & V107 (\%) & $23 \pm 15$ & $0.4 \pm 1.0$ & $0.1 \pm 0.3$ & $0 \pm 0$ \\
\hline & \multirow{2}{*}{ VMAT } & V95 (\%) & $97.3 \pm 1.5$ & $99.0 \pm 0.7$ & $99.5 \pm 0.5$ & $99.0 \pm 1.4$ \\
\hline & & V107 (\%) & $14 \pm 5$ & $0.6 \pm 1.1$ & $0.2 \pm 0.3$ & $0.1 \pm 0.3$ \\
\hline \multirow{4}{*}{ MAMA DERECHA } & \multirow{2}{*}{3 DCRT } & V95 (\%) & $97.6 \pm 1.2$ & $98.2 \pm 1.2$ & $97 \pm 4$ & $99.7 \pm 0.7$ \\
\hline & & V107 (\%) & $21 \pm 7$ & $2 \pm 3$ & $0.2 \pm 0.5$ & $0.00 \pm 0.01$ \\
\hline & \multirow{2}{*}{ VMAT } & V95 (\%) & $97.1 \pm 1.1$ & $98.1 \pm 1.2$ & $98.8 \pm 0.9$ & $99.1 \pm 1.1$ \\
\hline & & V107 (\%) & $16 \pm 10$ & $0.7 \pm 1.4$ & $1.3 \pm 1.6$ & $0.1 \pm 0.4$ \\
\hline
\end{tabular}

Tabla 6. Estadística de dosis absorbidas recibidas por el corazón en tratamientos de mama izquierda.

\begin{tabular}{c|c|c|c|c|c}
\multirow{2}{*}{} & \multicolumn{5}{|c}{ CORAŹ́N } \\
\cline { 2 - 6 } & $D_{\text {media }}$ (Gy) & $D_{2 \%}$ (Gy) & V4 (\%) & V8 (\%) & V16 (\%) \\
\hline 3 DCRT & $2.6 \pm 0.8$ & $26 \pm 11$ & $11 \pm 6$ & $4.8 \pm 1.9$ & $3.3 \pm 1.4$ \\
\hline VMAT & $4.8 \pm 0.9$ & $18 \pm 5$ & $46 \pm 18$ & $13 \pm 6$ & $2.8 \pm 1.4$ \\
\hline$p$ & $<0.001$ & 0.011 & $<0.001$ & $<0.001$ & 0.251
\end{tabular}

Tabla 7. Estadística de dosis absorbidas recibidas por la DA en tratamientos de mama izquierda.

\begin{tabular}{c|c|c}
\multirow{2}{*}{} & \multicolumn{2}{|c}{ ARTERIA DESCENDIENTE ANTERIOR } \\
\cline { 2 - 3 } & $\boldsymbol{D}_{\text {media }}$ (Gy) & $\boldsymbol{D}_{\max }$ (Gy) \\
\hline 3 DCRT & $8 \pm 5$ & $25 \pm 13$ \\
\hline VMAT & $9 \pm 4$ & $20 \pm 7$ \\
\hline$p$ & 0.141 & 0.346
\end{tabular}

boost alrededor del mismo. Sin embargo la variabilidad es muy alta, debido entre otras cosas a los distintos tamaños del PTV Boost de cada una de las pacientes, así que no es un resultado que proporcione una información relevante (fig. 3b).

Los criterios dosimétricos que se han evaluado en corazón, arteria DA y pulmones ipsilaterales se recogen en las tablas 6,7 y 8 . Los resultados obtenidos con ambas técnicas han sido comparados mediante un Test $U$ de Mann-Whitney, siendo el nivel de significancia 0.05. Por tanto, los parámetros dosimétricos en los que el test arroja valores de $p$ inferiores a 0.05 presentan una diferencia entre ambos grupos estadísticamente significativa.

Los valores de exceso de riesgo relativo, $\mathrm{CERR}_{9}$ años, $y$ el exceso de riesgo absoluto, $\mathrm{CEAR}_{9}$ años, de sufrir un
Tabla 8. Estadística de dosis absorbidas recibidas por el pulmón ipsilateral.

\begin{tabular}{c|c|c|c|c}
\multirow{2}{*}{} & \multicolumn{3}{|c}{ PULMÓN IPSILATERAL } \\
\cline { 2 - 5 } & \multicolumn{2}{|c|}{ MAMA IZQUIERDA } & \multicolumn{2}{c}{ MAMA DERECHA } \\
\cline { 2 - 5 } & $D_{\text {media }}$ (Gy) & $V 16(\%)$ & $D_{\text {media }}$ (Gy) & $V 16(\%)$ \\
\hline 3 DCRT & $9.5 \pm 1.2$ & $21 \pm 3$ & $9.8 \pm 1.3$ & $21 \pm 4$ \\
\hline VMAT & $7.9 \pm 1.6$ & $17 \pm 5$ & $8.2 \pm 0.9$ & $18 \pm 3$ \\
\hline$p$ & $<0.001$ & 0.002 & $<0.001$ & 0.002 \\
\hline
\end{tabular}

ACE en los 9 años posteriores a la radioterapia en pacientes con tratamiento de mama izquierda para las dos técnicas se muestran en la tabla 9. Se ha agrupado los resultados por década de edad de la paciente en el momento del tratamiento y por la existencia o ausencia de factores de riesgo de ACE previos respectivamente.

En la tabla 10 se recogen las OED calculadas para la mama contralateral y ambos pulmones con los distintos modelos, distinguiendo entre tratamientos de mama izquierda y de mama derecha. Dado que los valores que encontramos para ambas lateralidades son muy similares, en la tabla 11 se muestra el EAR calculado para mama contralateral y pulmones y el 
Tabla 9. $\mathrm{CERR}_{9 \text { años }}$ y $\mathrm{CEAR}_{9}$ años promedio en función de la técnica de irradiación, agrupados por edad o por la existencia de factores de riesgo previos al tratamiento.

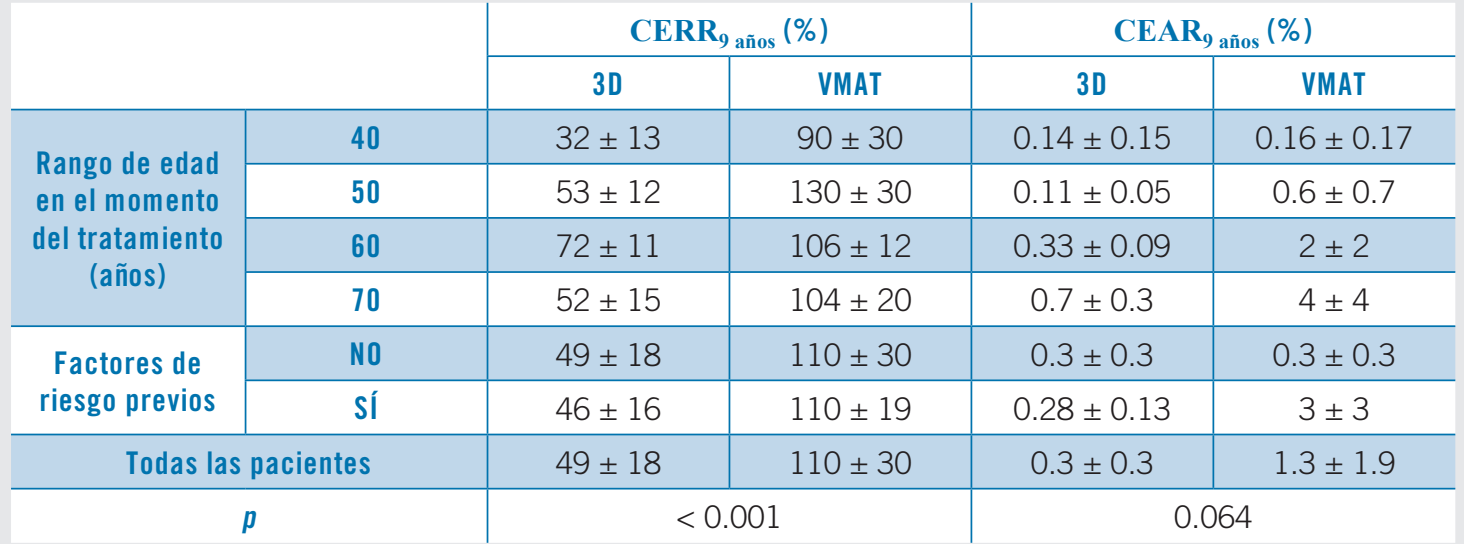

Tabla 10. OED (Gy) calculados para ambas lateralidades, técnicas y con los tres modelos descritos.

\begin{tabular}{|c|c|c|c|c|c|}
\hline & & & $\begin{array}{c}\text { OED Mama } \\
\text { Contralateral }\end{array}$ & $\begin{array}{l}\text { OED Pulmón } \\
\text { Contralateral }\end{array}$ & $\begin{array}{l}\text { OED Pulmón } \\
\text { Ipsilateral }\end{array}$ \\
\hline \multirow{6}{*}{ MAMA IZQUIERDA } & \multirow{3}{*}{3 DCRT } & L-E & $0.5 \pm 0.2$ & $0.54 \pm 0.14$ & $4.1 \pm 0.4$ \\
\hline & & Plateau & $0.5 \pm 0.2$ & $0.54 \pm 0.14$ & $4.1 \pm 0.4$ \\
\hline & & Mecánico & $0.5 \pm 0.2$ & $0.53 \pm 0.14$ & $4.1 \pm 0.4$ \\
\hline & \multirow{3}{*}{ VMAT } & L-E & $1.9 \pm 0.8$ & $2.0 \pm 0.3$ & $4.5 \pm 0.5$ \\
\hline & & Plateau & $1.8 \pm 0.7$ & $1.9 \pm 0.3$ & $4.4 \pm 0.4$ \\
\hline & & Mecánico & $1.9 \pm 0.8$ & $1.9 \pm 0.3$ & $4.4 \pm 0.4$ \\
\hline \multirow{6}{*}{ MAMA DERECHA } & \multirow{3}{*}{3 DCRT } & L-E & $0.5 \pm 0.2$ & $0.65 \pm 0.16$ & $4.3 \pm 0.4$ \\
\hline & & Plateau & $0.4 \pm 0.2$ & $0.64 \pm 0.16$ & $4.4 \pm 0.4$ \\
\hline & & Mecánico & $0.5 \pm 0.2$ & $0.63 \pm 0.15$ & $4.3 \pm 0.4$ \\
\hline & \multirow{3}{*}{ VMAT } & $L-E$ & $1.9 \pm 0.6$ & $1.7 \pm 0.4$ & $4.5 \pm 0.5$ \\
\hline & & Plateau & $1.8 \pm 0.6$ & $1.7 \pm 0.4$ & $4.4 \pm 0.4$ \\
\hline & & Mecánico & $1.9 \pm 0.6$ & $1.6 \pm 0.4$ & $4.3 \pm 0.4$ \\
\hline
\end{tabular}

Tabla 11. EARs expresado en casos por 10000 pacientes por año, promediando tratamientos de mama izquierda y derecha.

\begin{tabular}{c|c|c|c|c|c}
\multicolumn{2}{c|}{} & $\begin{array}{c}\text { EAR Mama } \\
\text { Contralateral }\end{array}$ & $\begin{array}{c}\text { EAR Pulmón } \\
\text { Contralateral }\end{array}$ & $\begin{array}{c}\text { EAR Pulmón } \\
\text { Ipsilateral }\end{array}$ & EAR Total \\
\hline \multirow{3}{*}{3 DCRT } & L-E & $3.9 \pm 1.2$ & $4.8 \pm 0.9$ & $34 \pm 2$ & $42 \pm 3$ \\
\cline { 2 - 6 } & Plateau & $3.8 \pm 1.2$ & $4.7 \pm 0.9$ & $34 \pm 2$ & $44 \pm 2$ \\
\cline { 2 - 6 } & Mecánico & $3.9 \pm 1.2$ & $4.6 \pm 0.8$ & $34 \pm 2$ & $43 \pm 3$ \\
\hline \multirow{3}{*}{ VMAT } & L-E & $16 \pm 4$ & $14.6 \pm 1.9$ & $36 \pm 3$ & $66 \pm 5$ \\
\cline { 2 - 6 } & Plateau & $15 \pm 4$ & $14.3 \pm 1.8$ & $35 \pm 3$ & $64 \pm 3$ \\
\cline { 2 - 6 } & Mecánico & $15 \pm 4$ & $13.9 \pm 1.8$ & $35 \pm 2$ & $64 \pm 5$
\end{tabular}


Tabla 12. Niveles de significancia obtenidos con el Test $U$ de Mann-Whitney para los conjuntos de datos de EAR calculados en tratamientos con 3DCRT y VMAT.

\begin{tabular}{c|c|c|c|c|c}
\multicolumn{2}{c|}{} & $\begin{array}{c}\text { EAR Mama } \\
\text { Contralateral }\end{array}$ & $\begin{array}{c}\text { EAR Pulmón } \\
\text { Contralateral }\end{array}$ & $\begin{array}{c}\text { EAR Pulmón } \\
\text { Ipsilateral }\end{array}$ & EAR Total \\
\hline \multirow{3}{*}{$p$} & L-E & $<0.001$ & $<0.001$ & 0.004 & $<0.001$ \\
\cline { 2 - 7 } & Plateau & $<0.001$ & $<0.001$ & 0.196 & $<0.001$ \\
\cline { 2 - 7 } & Mecánico & $<0.001$ & $<0.001$ & 0.607 & $<0.001$
\end{tabular}

EAR total, considerando en conjunto los tratamientos de mama izquierda y derecha.

\section{Discusión}

La cardiotoxicidad asociada a los tratamientos con radioterapia es un asunto controvertido. La mayoría de las pacientes con cáncer de mama que requieren radioterapia en las cadenas ganglionares también precisan quimioterapia, que generalmente incluye en sus esquemas agentes con conocidos efectos cardiotóxicos como las antraciclinas. ${ }^{26}$ Además habitualmente estos tratamientos se combinan con la administración de anticuerpos monoclonales como el Trastuzumab (Herceptín ${ }^{\circledR}$ ) que también contribuyen a la toxicidad cardíaca, especialmente si se administran de forma concomitante $^{27}$ o la paciente presenta factores de riesgo previos como un índice de masa corporal elevado. ${ }^{28,29}$ Por todo ello, es complicado predecir a largo plazo qué parte de los efectos cardiacos son achacables a un tratamiento u otro.

Darby et al., ${ }^{25}$ en 2013 relacionaban de manera lineal la dosis absorbida media recibida por el corazón con el riesgo de sufrir un ACE. En este estudio, basado en tratamientos de mama de 2168 mujeres entre 1958-2001 en Suecia y Dinamarca, encentraban un aumento relativo de la incidencia acumulada de ACE (CERR) del $7.4 \%$ por cada Gy de dosis absorbida media que recibe el corazón, siendo este porcentaje de aumento similar para mujeres con y sin factores de riesgo previos. Además encontraban que el mayor incremento del riesgo se produce en los primeros años tras la radioterapia, con CERR $=16.3 \%$ por Gy en los primeros 4 años post tratamiento y $15.5 \%$ por Gy en los siguientes 5-9 años) y que éste continúa hasta pasados más de 20 años tras la irradiación $(8.2 \%$ por Gy de 20 años en adelante). El estudio de Darby cuenta con una limitación importante, ya que gran parte de las pacientes incluidas en el mismo fueron en realidad tratamientos 2D, en los que no se realizaba un TAC de simulación, y por tanto la evaluación de la dosis impartida a órganos de riesgo fue realizada a posteriori con una reconstrucción de los haces sobre un TAC de paciente con anatomía estándar.
Otros estudios posteriores al de Darby devuelven resultados en la misma línea, encontrando por ejemplo que la incidencia relativa de infarto de miocardio aumenta linealmente un $6.4 \%$ por Gy de dosis absorbida media al corazón, incluso que dicha respuesta no se modifica significativamente con los porcentajes de dosis-volumen que recibe el corazón completo (Jacobse et al. ${ }^{30}$ ), o que existe un aumento de la mortalidad debida a eventos cardiacos de $0.04 \%$ por Gy a 10 años (Taylor et al. ${ }^{31}$ )

Posteriormente Bogaard et al., ${ }^{24}$ en un estudio en 2017 validaban los resultados de Darby con un conjunto independiente de pacientes, en las cuales se disponía ya de CT y de distribución de dosis tridimensional. Para ello utilizan un modelo predictivo en el cual incluyen, además de la dosis media, la edad y factores de riesgo para ACE previos a la radioterapia. Éste modelo es el que hemos utilizado en este trabajo para cuantificar los posibles daños producidos al corazón. En dicho estudio encontraban un aumento promedio relativo del $16.5 \%$ en la incidencia acumulada de ACE por Gy de dosis media absorbida por el corazón en los primeros 9 años tras el tratamiento, lo cual es consistente con los valores proporcionados por Darby.

En nuestro trabajo encontramos que la dosis absorbida alta que recibe el corazón, evaluada como el valor que recibe el $2 \%$ del volumen $\left(D_{2} \%\right)$ se reduce en casi 10 Gy con VMAT, con $p<0.011$. Sin embargo la dosis absorbida media del mismo aumenta en 2.2 Gy ( $p<$ 0.001 ), ya que hay un aumento del volumen de corazón que recibe dosis absorbidas bajas, como se puede ver reflejado tanto en el V4 $(p<0.001)$ como en el V8 $(p<0.001)$. La aparente disminución del volumen alcanzado con 16 Gy en los casos con VMAT no alcanza en cambio niveles significativos (fig. 4).

En la tabla 9 vemos que el valor promedio total del exceso de riesgo relativo, $\mathrm{CERR}_{9}$ años, que obtenemos es bastante superior con VMAT (110\%) que con 3DCRT (49\%) con $p<0.001$, lo cual concuerda razonablemente bien con los resultados presentados por Bogaard, que encontraba un aumento promedio del $\mathrm{CERR}_{9}$ años de $16.6 \%$ por Gy de dosis absorbida media. El valor del exceso de riesgo absoluto, $\mathrm{CEAR}_{9}$ años, aumenta de $0.3 \%$ a $1.3 \%$ al utilizar VMAT (Bogaard encontraba $1.13 \%)$, lo cual implicaría que de cada 100 pacientes 

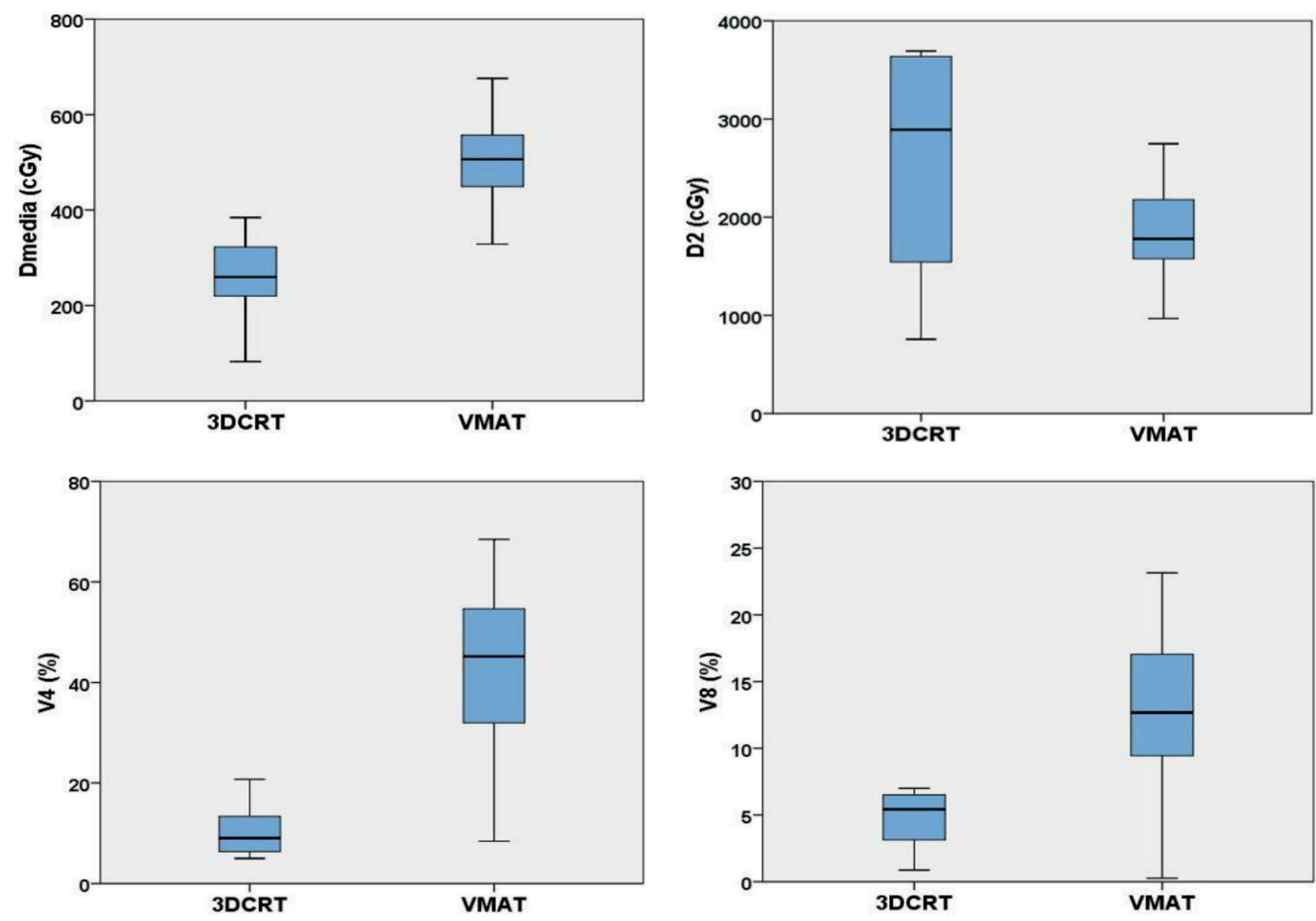

Fig. 4. Representación gráfica de los parámetros dosimétricos de corazón en los que la diferencia entre las dos técnicas de irradiación es estadísticamente significativa.

tratadas con radioterapia con VMAT, una de ellas sufriría un ACE debido a la radiación en los siguientes 9 años que no hubiera sufrido de haberse tratado con 3DCRT. Sin embargo no podemos decir que este aumento sea estadísticamente significativo ( $p=0.064)$.

Pese que los resultados son consistentes con lo que encontramos en la literatura, tenemos que la dispersión de resultados es muy elevada, como podemos ver en la fig. 5, ya que depende de forma muy importante tanto de la edad de la paciente como de la existencia de factores de riesgo previos, y el número de pacientes que tenemos en cada uno de los subgrupos no es suficiente para obtener resultados significativos de cada sector.

Cabe destacar que, pese a que los resultados de Bogaard ratifican los hallazgos iniciales de Darby y por tanto existencia de una dependencia de los AEC con la dosis media recibida por el corazón, ambos trabajos mantienen una limitación importante que es que se basan en distribuciones de dosis 3DCRT, muy inhomogéneas en el corazón. Es decir los valores de dosis media corresponden con dosis altas en la parte más cercana a la mama y prácticamente dosis nula en el resto. No hay hasta la fecha, hasta donde llega nues- tro conocimiento, evidencia de que esa relación sea extensible directamente a una distribución de dosis más homogénea pero de valores más bajos.

Por otra parte, diversos autores hacen hincapié en el hecho de que, dado que la morbilidad predominante es la cardiopatía isquémica, pueden ser las arterias coronarias, y en concreto la DA, las estructuras más críticas a la hora de inducir morbilidad coronaria. ${ }^{32}$ En este caso se preguntan si la dosis absorbida media al corazón es un parámetro a tener en cuenta, ya que no refleja la heterogeneidad de la irradiación y los posibles puntos calientes de dosis absorbida que pueda haber en estructuras críticas, como la DA. En esta línea, Correa et al., 33 realizaron un estudio sobre el aumento en las anormalidades reveladas en un test de estrés realizado a pacientes 12 años tras el tratamiento frente a pacientes no tratadas. El 59\% en las pacientes de mama izquierda presentaban anormalidades en el test mientras que solamente aparecían en un $8 \%$ de las de mama derecha. Además el 70\% de dichas anormalidades se encontraba en la zona de la arteria descendente anterior, por lo que concluyen que técnicas modernas de irradiación, como la VMAT, podrían reducir este riesgo de daño cardiaco. 

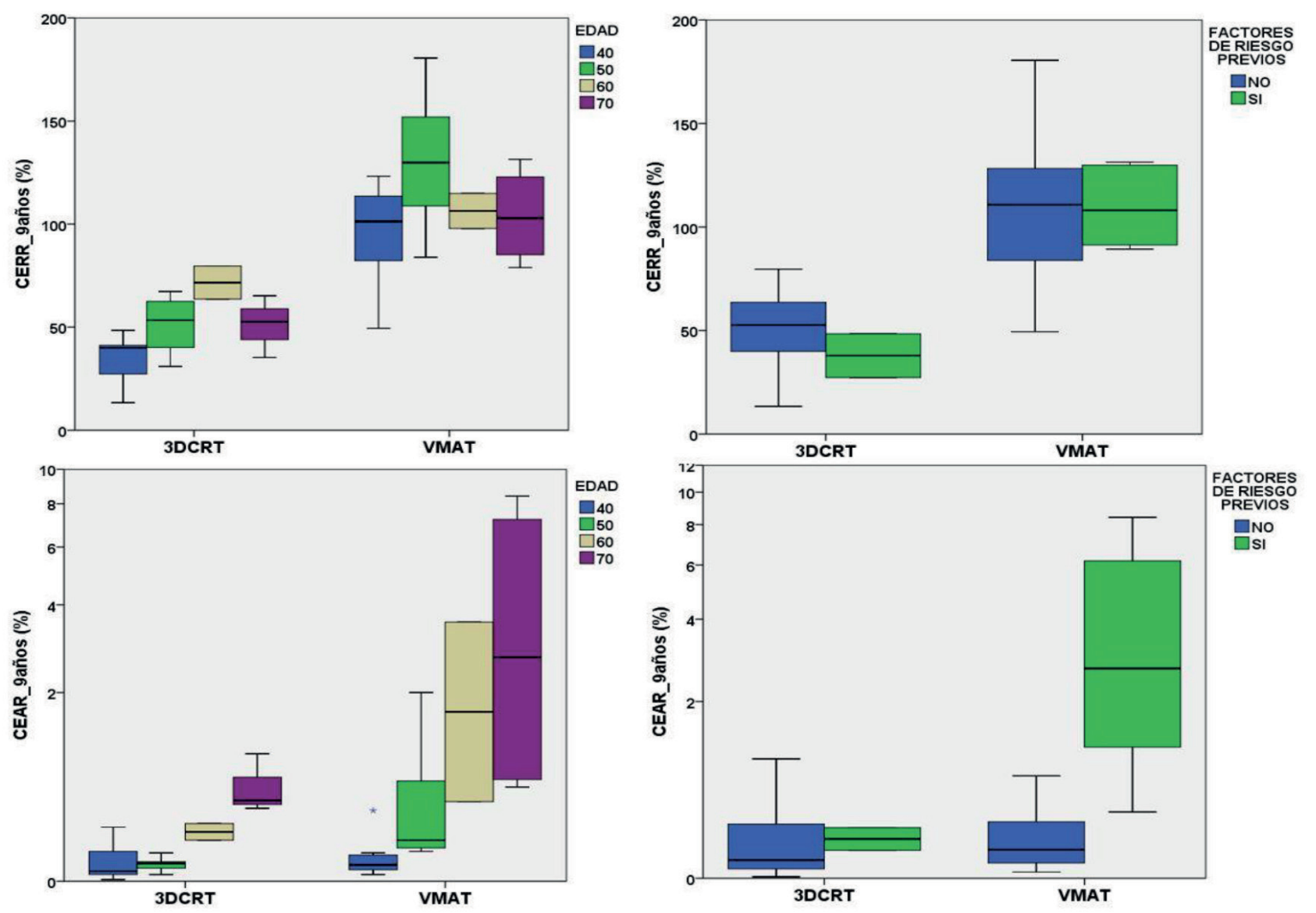

Fig. 5. $\mathrm{CERR}_{9}$ años y $_{\mathrm{CEAR}}$ años en \% representados en función de la técnica de irradiación, agrupados por edad en el momento del tratamiento y por la existencia o no de factores de riesgo previos al tratamiento.

Algunos autores ${ }^{34,35}$ establecen una correlación entre los parámetros dosimétricos del corazón y los de la DA, llegando incluso a asegurar que no es necesaria su delimitación pese a no negar su importancia. Sin embargo esta correlación solamente sería válida de nuevo para el caso de planificaciones 3DCRT con haces tangenciales, ya que está basada en la geometría de la irradiación. Otros en cambio no encuentran dicha correlación y concluyen que para minimizar el riesgo de eventos cardiovasculares es necesaria la evaluación tanto de la dosis absorbida media al corazón como de la dosis absorbida media a la DA. ${ }^{36}$

A día de hoy no existen recomendaciones de consenso sobre la limitación de dosis que se debe aplicar en la DA. En 2009 el grupo DBCG (Danish Breast Cancer Cooperative Group) recomendó una $\mathrm{D}_{\max }<20 \mathrm{~Gy}, 32$ en otros trabajos se habla de valores críticos de $\mathrm{D}_{\text {media }}<19$ Gy ${ }^{35}$ o V20 < 10\%. ${ }^{36}$ Estos límites no solamente no están estandarizados sino que además deben leerse con precaución, ya que los criterios de delimitación de la DA no son únicos.

Nuestros resultados en éste órgano corresponden con una disminución en la dosis absorbida máxima de
5 Gy (de 25 a 20 Gy) y un valor muy similar para ambas técnicas de la dosis absorbida media (8-9 Gy), sin embargo no se encontró en ninguno de los dos casos que la dependencia con la técnica fuera significativa (tabla 7). En todo caso, como ya se ha comentado previamente, la DA se delimitó en nuestras pacientes a posteriori, y por tanto no fue tenida en cuenta como órgano de riesgo en la optimización. Considerando el hecho de que en nuestras pacientes encontramos con VMAT una disminución promedio de casi de 10 Gy en el $\mathrm{D}_{2 \%}$, del corazón, es decir, una dosis inferior en la zona más cercana a la mama que precisamente la zona en la que se encuentra la DA, parece razonable pensar que en no sería complicado reducir la dosis en la DA en el momento que se incluya en el proceso de optimización.

El otro órgano de riesgo en el que la variación de la distribución de dosis absorbida es relevante es el pulmón ipsilateral. Al igual que ocurre en el corazón, pasamos de tener una distribución en la que gran parte del pulmón recibe una dosis absorbida bastante alta a una distribución con menos dosis absorbidas altas pero en la que prácticamente todo el pulmón recibe dosis absorbidas bajas. 
La radiotoxicidad más frecuente en el pulmón es la neumonitis rádica, una toxicidad considerada como temprana-intermedia, que se desarrolla entre 6 a 21 semanas tras el tratamiento y que ocasionalmente puede acabar en una fibrosis pulmonar. Su incidencia sin embargo está muy influenciada por factores externos a la radioterapia, como la administración de quimioterapia, edad, o hábitos de la paciente como el tabaquismo. Al igual que ocurre con la cardiotoxicidad, a la hora de evaluar la toxicidad pulmonar hay otros factores implicados en el tratamiento, como por ejemplo el empleo de fármacos como los taxanos, que tienen un relación conocida con la aparición de neumonitis, ${ }^{37,38}$ y que hacen que no sea inmediato establecer una relación causa efecto entre neumonitis y dosis de radiación absorbida en los pulmones.

El porcentaje de pulmón irradiado con dosis absorbidas intermedias (V20 a fraccionamiento convencional) y la dosis absorbida media de pulmón son los parámetros dosimétricos que mayor correlación guardan con la toxicidad pulmonar, tanto clínica, como radiológica y fisiológica. ${ }^{39,40}$ Goldman et al. ${ }^{41}$ afirman no encontrar ningún caso de neumonitis sintomática en pacientes cuyo V20 < 30\% y que el V13 es el parámetro mejor correlacionado con cambios radiológicos en el CT y a su vez con la dosis absorbida media del pulmón.

En nuestro estudio nos encontramos que tanto la $D_{\text {media }}$ como el V16 del pulmón ipsilateral (equivalente al V20 de fraccionamiento convencional) se reduce de forma significativa en los tratamientos con VMAT (tabla 8). En ambas lateralidades la dosis absorbida media es 1.6 Gy inferior que en los tratamientos 3DCRT $(p<0.001)$ y el V16 disminuye un $4.3 \%$ en mama izquierda y un $3.3 \%$ en mama derecha $(p<0.002)$. Por lo tanto la probabilidad de desarrollar neumonitis radioinducida, tanto clínica como radiológica, se vería reducida al realizar los tratamientos de mama con VMAT

Dado que los resultados encontrados en cuanto a estimación del riesgo de desarrollar tumores sólidos radioinducidos son similares con los tres modelos

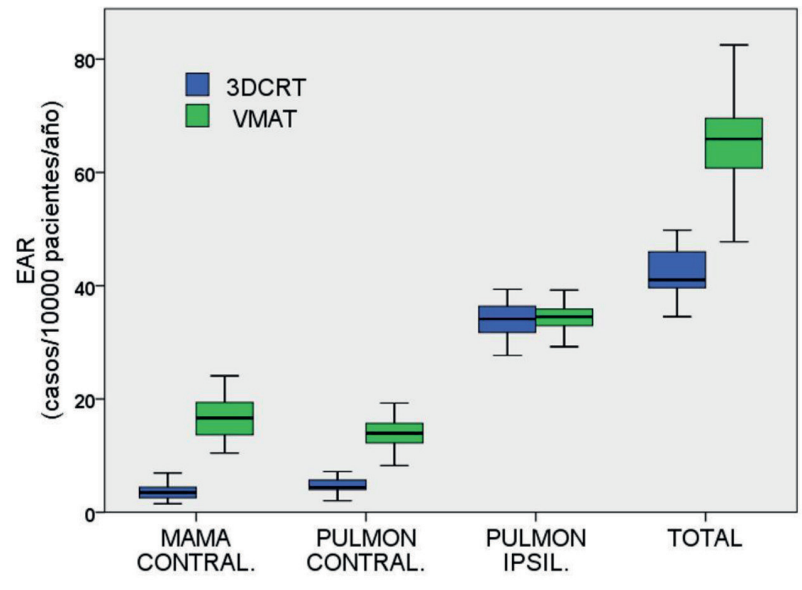

Fig. 6. Exceso Absoluto de Riesgo de cáncer secundario calculado con el modelo mecánico para los distintos órganos de riesgo y técnicas de irradiación.

de OED utilizados (tabla 11), por simplicidad vamos a centrar la discusión en los proporcionados por el modelo mecánico, ya que es el más completo.

EI EAR total calculado para las pacientes tratadas con VMAT es significativamente mayor $(p<0.001)$ que en tratamientos 3DCRT. Si evaluamos en conjunto los resultados de ambas lateralidades tenemos que con VMAT el EAR total sufre un aumento de 0.21 puntos porcentuales (p.p.) es decir, de cada 10000 mujeres tratadas con radioterapia a los 30 años, a la edad de 70 los casos de tumor sólido radioinducido por año serían 21 más si se irradiaron con VMAT que con 3DCRT (fig. 6). Este aumento se produce por la contribución de las dosis absorbidas bajas a la OED de mama y pulmón contralaterales. EI EAR en la mama contralateral con VMAT aumenta 0.11 p.p., mientras que el EAR del pulmón contralateral lo haría 0.09 p.p. En cambio la EAR del pulmón ipsilateral se mantiene en valores muy similares para ambas técnicas.

Si nos fijamos en los valores publicados en la literatura encontramos un rango bastante amplio de valores (tabla 13).

Tabla 13. Valores de EAR, estimados con el modelo mecánico, encontrados en la literatura para tratamientos de mama.

\begin{tabular}{c|c|c|c|c|c|c} 
& \multicolumn{2}{|c|}{$\begin{array}{c}\text { Mama } \\
\text { Contralateral }\end{array}$} & \multicolumn{2}{c|}{$\begin{array}{c}\text { Pulmón } \\
\text { Contralateral }\end{array}$} & \multicolumn{2}{c}{$\begin{array}{c}\text { Pulmón } \\
\text { Ipsilateral }\end{array}$} \\
\cline { 2 - 7 } & 3DCRT $\left(^{*}\right)$ & VMAT & 3DCRT $\left.{ }^{*}\right)$ & VMAT & 3DCRT (*) & VMAT \\
\hline Fogliata & $1.7 \pm 0.4$ & $8.5 \pm 1.4$ & $1.4 \pm 0.4$ & $7.3 \pm 1.3$ & $20 \pm 5$ & $22 \pm 3$ \\
\hline Karpf & $3 \pm 2$ & $5 \pm 2$ & $7 \pm 1$ & $12 \pm 2$ & $27 \pm 2$ & $27 \pm 2$ \\
\hline Haciislamoglu & $4.4 \pm 0.7$ & $20 \pm 2$ & $3.5 \pm 0.6$ & $22 \pm 3$ & $28 \pm 8$ & $65 \pm 5$ \\
\hline Este trabajo & $3.9 \pm 2$ & $15 \pm 4$ & $4.6 \pm 0.8$ & $13.9 \pm 1.8$ & $34 \pm 2$ & $35 \pm 2$
\end{tabular}

$\left(^{*}\right)$ Karpf no estima los valores para 3DCRT sino para IMRT tangencial con 5 -7 haces. 
Los trabajos de Fogliata y Karpf estiman el EAR para pacientes de mama izquierda, sin inclusión de cadenas ganglionares, tratadas en inspiración mantenida. Fogliata utiliza un esquema de tratamiento hipofraccionado (40.05 Gy en 15 fracciones) mientras que Karpf sigue un esquema tradicional (50.4 Gy en 28 fracciones). Los valores que presentan para pulmones son compatibles con los que obtenemos en este trabajo, teniendo en cuenta que al incluir cadenas ganglionares el volumen de pulmón irradiado aumenta. Sin embargo en mama los resultados de ambos son bastante inferiores a los nuestros, probablemente debido a que los coeficientes $\alpha$ y R que utilizan para mama están extraídos de ${ }^{23}$ mientras que para pulmón utilizan los que publicó posteriormente el mismo Schneider en ${ }^{20}$, que son los que se han utilizado en este trabajo como reflejábamos en la tabla 3.

Por otra parte Haciislamoglu et al. ${ }^{42}$ evalúan tratamientos de mama izquierda, sin inclusión de cadenas ganglionares, sin control respiratorio y con un esquema de 50 Gy en 25 fracciones. Los parámetros que utilizan para el cálculo en este caso son los mismos que los nuestros y los valores obtenidos para mama contralateral y pulmón contralateral son muy similares a los presentados con 3DCRT y algo superiores con VMAT. Sin embargo el valor que obtienen en el pulmón ipsilateral con VMAT es muy superior al resto de valores publicados en la literatura y al nuestro, sin que encontremos ningún indicio en su artículo que nos permita explicar este hecho.

Por último, debemos destacar que existe un sesgo intrínseco en los resultados obtenidos en la comparación de planificaciones de nuestros centros, ya que precisamente son los pacientes en los que la anatomía era más desfavorable para corazón y/o pulmón ipsilateral las que se planificaron con VMAT, lo cual puede influir negativamente en los valores de dosis absorbida encontrados en estos órganos.

\section{Conclusiones}

Los tratamientos con VMAT permiten, manteniendo niveles de cobertura y sobredosificaciones similares a los que tenemos con 3DCRT, disminuir las dosis absorbidas medias-altas que alcanzan el corazón y el pulmón ipsilateral. Esto permite, en los casos en los que la dosimetría 3DCRT no se ajusta a los límites de OAR establecidos, proporcionar un tratamiento radioterápico adecuado a las pacientes con cáncer de mama. Sin embargo, no recomendamos considerar la VMAT como tratamiento estándar para todas las pacientes, ya que la irradiación de órganos circundantes con dosis absorbidas bajas aumenta el riesgo de ciertas complicaciones cardíacas y de formación de tumores radioinducidos.
En caso de utilizar VMAT en pacientes de avanzada edad, especialmente si presentan factores de riesgo cardiovasculares previos a la irradiación, hay que ser muy cuidadoso con la dosis que alcanza el corazón, ya que es en estas pacientes en las que el exceso absoluto de riesgo de ACE como consecuencia de la irradiación es más importante. Sin embargo en estos casos la probabilidad de aparición de tumores radioinducidos no debe considerarse un factor limitante a la hora de realizar la dosimetría. Por el contrario en pacientes jóvenes y sin factores de riesgo cardiovasculares previos, el aumento del riesgo absoluto de ACE por el uso de VMAT frente 3DCRT es muy pequeño y se debe prestar más atención a reducir el baño de dosis bajas en pulmones y mama contralateral para reducir al máximo la probabilidad de desarrollar tumores secundarios radioinducidos en los años venideros.

Por todo ello, a la hora de utilizar VMAT como técnica de irradiación, recomendamos evaluar la dosis absorbida media en corazón y asegurarse de que se mantiene en valores similares a los niveles de referencia que tendríamos en caso de una irradiación 3DCRT. Además se recomienda minimizar la dosis absorbida recibida por arteria descendiente anterior, pese a no existir actualmente un consenso sobre el límite máximo a fijar.

\section{Referencias}

1. Lee B, Lee S, Sung J, Yoon M. Radiotherapy-induced secondary cancer risk for breast cancer: 3D conformal therapy versus IMRT versus VMAT. J Radiol Prot. 2014;34(2):325-31.

2. Bogue J, Wan J, Lavey RS, Parsai El. Dosimetric comparison of VMAT with integrated skin flash to 3D field-in-field tangents for left breast irradiation. J Appl Clin Med Phys. 2019;20(2):24-9.

3. Byrne M, Archibald-Heeren B, Hu Y, Fong A, Chong L, Teh A. Comparison of semiautomated tangential VMAT with 3DCRT for breast or chest wall and regional nodes. J Appl Clin Med Phys. 2018;19(5):684-93.

4. Pignol JP, Truong P, Rakovitch E, Sattler MG, Whelan TJ, Olivotto IA. Ten years results of the Canadian breast intensity modulated radiation therapy (IMRT) randomized controlled trial. Radiother Oncol. 2016 Dec 1;121(3):414-9.

5. Jensen KE, Soril LJJ, Stelfox HT, Clement FM, Lin Y, Marshall DA. Side Effects Associated with the Use of IntensityModulated Radiation Therapy in Breast Cancer Patients Undergoing Adjuvant Radiation Therapy: A Systematic Review and Meta-Analysis. J Med Imaging Radiat Sci [Internet]. 2017;48(4):402-13. Available from: https://doi. org/10.1016/j.jmir.2017.09.002

6. Marta GN, Hanna SA, Martella E, Silva JLF da. Complications from radiotherapy for breast cancer. Sao Paulo Med J. 2011;129(2):116-7.

7. Sloan L, Alcorn S. Acute and Late Skin Toxicity from Breast Radiation. In: Wright J., editor. Toxicities of Radiation Treatment for Breast Cancer. Springer, Cham; 2019. p. 5-22. 
8. Willner J, Jost A, Baier K, Flentje M. A little to a lot or a lot to a little? An analysis of pneumonitis risk from dose-volume histogram parameters of the lung in patients with lung cancer treated with 3-D conformal radiotherapy. Strahlentherapie und Onkol. 2003;179(8):548-56.

9. Chao PJ, Lee HF, Lan JH, Guo SS, Ting HM, Huang YJ, et al. Propensity-score-matched evaluation of the incidence of radiation pneumonitis and secondary cancer risk for breast cancer patients treated with IMRT/NMAT. Sci Rep [Internet]. 2017;7(1):1-9. Available from: http://dx.doi.org/10.1038/ s41598-017-14145-x

10. Liu H, Chen X, He Z, Li J. Evaluation of 3D-CRT, IMRT and VMAT radiotherapy plans for left breast cancer based on clinical dosimetric study. Comput Med Imaging Graph [Internet]. 2016;54:1-5. Available from: http://dx.doi.org/10.1016/j. compmedimag.2016.10.001

11. Allemani C, Sant M, Weir HK, Richardson LC, Baili P, Storm $\mathrm{H}$, et al. Breast cancer survival in the US and Europe: a CONCORD high- resolution study. Int Jo. 2013;132(5):117081.

12. American Cancer Society. Breast Cancer Facts \& Figures 2019-2020. Atlanta: American Cancer Society, Inc. 2019.

13. Andersen ER, Eilertsen G, Myklebust AM, Eriksen S. Women's experience of acute skin toxicity following radiation therapy in breast cancer. J Multidiscip Healthc. 2018;11:139-48.

14. Shapiro CL, Recht. M. Side Effects o Adjuvant Tratment of Breast Cancer. N Engl J Med. 2001;344(26):1997-2008.

15. Brownlee Z, Garg R, Listo M, Zavitsanos P, Wazer DE, Huber $\mathrm{KE}$. Late complications of radiation therapy for breast cancer: Evolution in techniques and risk over time. Gland Surg. 2018;7(4):371-8.

16. Schneider U, Zwahlen D, Ross D, Kaser-Hotz B. Estimation of radiation-induced cancer from three-dimensional dose distributions: Concept of organ equivalent dose. Int J Radiat Oncol Biol Phys. 2005;61(5):1510-5.

17. Abo-Madyan Y, Aziz MH, Aly MMOM, Schneider F, Sperk E, Clausen S, et al. Second cancer risk after 3D-CRT, IMRT and VMAT for breast cancer. Radiother Oncol [Internet]. 2014;110(3):471-6. Available from: http://dx.doi. org/10.1016/j.radonc.2013.12.002

18. Zhang $\mathrm{Q}$, Liu J, Ao ningjian, Yu $\mathrm{H}$, peng $\mathrm{Y}$, ou $\mathrm{L}$, et al. Secondary cancer risk after radiation therapy for breast cancer with different radiotherapy techniques. 2020;

19. Preston DL, Ron E, Tokuoka S, Funamoto S, Nishi N, Soda $M$, et al. Solid Cancer Incidence in Atomic Bomb Survivors: 1958-1998. Radiat Res. 2007;168(1):1-64.

20. Schneider U, Sumila M, Robotka J. Site-specific dose-response relationships for cancer induction from the combined Japanese A-bomb and Hodgkin cohorts for doses relevant to radiotherapy. Theor Biol Med Model. 2011;8(1):1-21.

21. Schneider U. Mechanistic model of radiation-induced cancer after fractionated radiotherapy using the linear-quadratic formula. Med Phys. 2009;36(4):1138-43.

22. Schneider U, Stipper A, Besserer J. Dose-response relationship for lung cancer induction at radiotherapy dose. Z Med Phys. 2010;20(3):206-14.

23. Schneider U, Sumila M, Robotka J, Gruber G, Mack A, Besserer J. Dose-response relationship for breast cancer induction at radiotherapy dose. Radiat Oncol. 2011;6(1):1-7.

24. Van Den Bogaard VAB, Ta BDP, Van Der Schaaf A, Bouma $\mathrm{AB}$, Middag AMH, Bantema-Joppe EJ, et al. Validation and modification of a prediction model for acute cardiac events in patients with breast cancer treated with radiotherapy based on three-dimensional dose distributions to cardiac substructures. J Clin Oncol. 2017;35(11):1171-8.

25. Darby SC, Ewertz M, McGale P, Bennet AM, Blom-Goldman $U$, Brnønum D, et al. Risk of ischemic heart disease in women after radiotherapy for breast cancer. N Engl J Med. 2013;368(11):987-98.

26. Cai $F$, Luis MAF, Lin X, Wang M, Cai L, Cen C, et al. Anthracycline-induced cardiotoxicity in the chemotherapy treatment of breast cancer: Preventive strategies and treatment. Mol Clin Oncol. 2019;11(1):15-23.

27. Nicolazzi MA, Carnicelli A, Fuorlo M, Scaldaferri A, Masetti $\mathrm{R}$, Landolfi R, et al. Anthracycline and trastuzumab-induced cardiotoxicity in breast cancer. Eur Rev Med Pharmacol Sci. 2018;22(7):2175-85.

28. Kaboré EG, Guenancia C, Vaz-Luis I, Di Meglio A, Pistilli $B$, Coutant $C$, et al. Association of body mass index and cardiotoxicity related to anthracyclines and trastuzumab in early breast cancer: French CANTO cohort study. PLoS Med. 2019;16(12):1-12.

29. Guenancia C, Lefebvre A, Cardinale D, Yu AF, Ladoire S, Ghiringhelli $F$, et al. Obesity as a risk factor for anthracyclines and trastuzumab cardiotoxicity in breast cancer: A systematic review and meta-analysis. J Clin Oncol. 2016;34(26):3157-65.

30. Jacobse JN, Duane FK, Boekel NB, Schaapveld M, Hauptmann M, Hooning MJ, et al. Radiation Dose-Response for Risk of Myocardial Infarction in Breast Cancer Survivors. Int J Radiat Oncol Biol Phys [Internet]. 2019;103(3):595-604. Available from: https://doi.org/10.1016/j.ijrobp.2018.10.025

31. Taylor C, Duane FK, Dodwell D, Gray R, Wang Z, Wang Y, et al. Estimating the Risks of Breast cancer radiotherapy: Evidence from modern radiation doses to the lungs and Heart and From previous randomized trials. J Clin Oncol. 2017;35(15):1641-9.

32. Offersen B, Højris I, Overgaard M. Radiation-induced heart morbidity after adjuvant radiotherapy of early breast cancer - Is it still an issue? Radiother Oncol [Internet]. 2011;100(2):157-9. Available from: http://dx.doi. org/10.1016/j.radonc.2011.08.007

33. Correa CR, Litt HI, Hwang WT, Ferrari VA, Solin LJ, Harris EE. Coronary artery findings after left-sided compared with right-sided radiation treatment for early-stage breast cancer. J Clin Oncol. 2007;25(21):3031-7.

34. Evans SB, Panigrahi B, Northrup V, Patterson J, Baldwin DE, Higgins SA, et al. Analysis of coronary artery dosimetry in the 3-dimensional era: Implications for organ-at-risk segmentation and dose tolerances in left-sided tangential breast radiation. Pract Radiat Oncol [Internet]. 2013;3(2):e55-60. Available from: http://dx.doi.org/10.1016/j.prro.2012.06.007

35. Becker-Schiebe M, Stockhammer M, Hoffmann W, Wetzel F, Franz $\mathrm{H}$. Does mean heart dose sufficiently reflect coronary artery exposure in left-sided breast cancer radiotherapy? Strahlentherapie und Onkol. 2016;192(9):624-31.

36. Plieskienè A, Burdulis D. Assessment Of The Dose To The Heart And The Left Anterior Descending Coronary Artery For The Left Breast Radiotherapy. Sveik Moksl. 2016;26(5):79_ 83.

37. Bielopolski D, Evron E, Moreh-Rahav O, Landes M, Stemmer SM, Salamon F. Paclitaxel-induced pneumonitis in patients with breast cancer: case series and review of the literature. J Chemother. 2017;29(2):113-7. 
38. Taghian AG, Assaad SI, Niemierko A, Kuter I, Younger $\mathrm{J}$, Schoenthaler $\mathrm{R}$, et al. Risk of pneumonitis in breast cancer patients treated with radiation therapy and combination chemotherapy with paclitaxel. J Natl Cancer Inst. 2001;93(23):1806-11.

39. Vasiljevic D, Arnold C, Neuman D, Fink K, Popovscaia M, Kvitsaridze I, et al. Occurrence of pneumonitis following radiotherapy of breast cancer - A prospective study. Strahlentherapie und Onkol. 2018;194(6):520-32.

40. Lind PA, Wennberg B, Gagliardi G, Rosfors S, Blom-Goldman $U$, Lideståhl $A$, et al. ROC curves and evaluation of radiation- induced pulmonary toxicity in breast cancer. Int J Radiat Oncol Biol Phys. 2006;64(3):765-70.

41. Goldman UB, Wennberg B, Svane G, Bylund H, Lind P. Reduction of radiation pneumonitis by $\mathrm{V} 20$-constraints in breast cancer. Radiat Oncol. 2010;5(1):1-6.

42. Haciislamoglu E, Cinar Y, Gurcan F, Canyilmaz E, Gungor G, Yoney $A$. Secondary cancer risk after whole-breast radiation therapy: Field-in-field versus intensity modulated radiation therapy versus volumetric modulated arc therapy. $\mathrm{Br} \mathrm{J}$ Radiol. 2019;92(1102). 


\section{BRAINLAB}

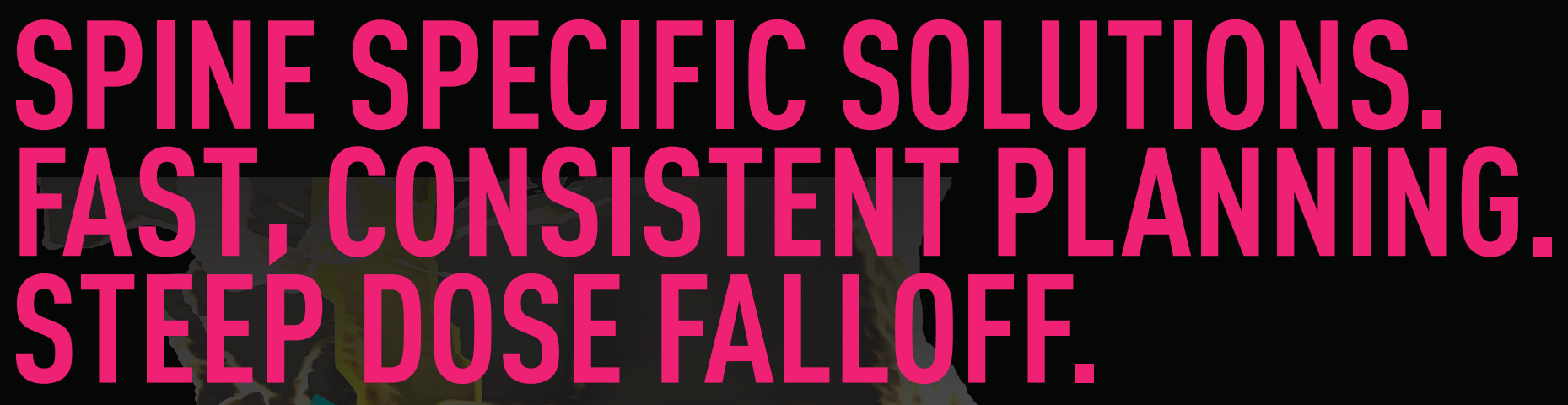

LEARN HOW BRAINLAB IS SHIFTING THE PARADIGM IN THE MANAGEMENT OF SPINAL LESIONS AT BRAINEAB. COM/SPINESRS.

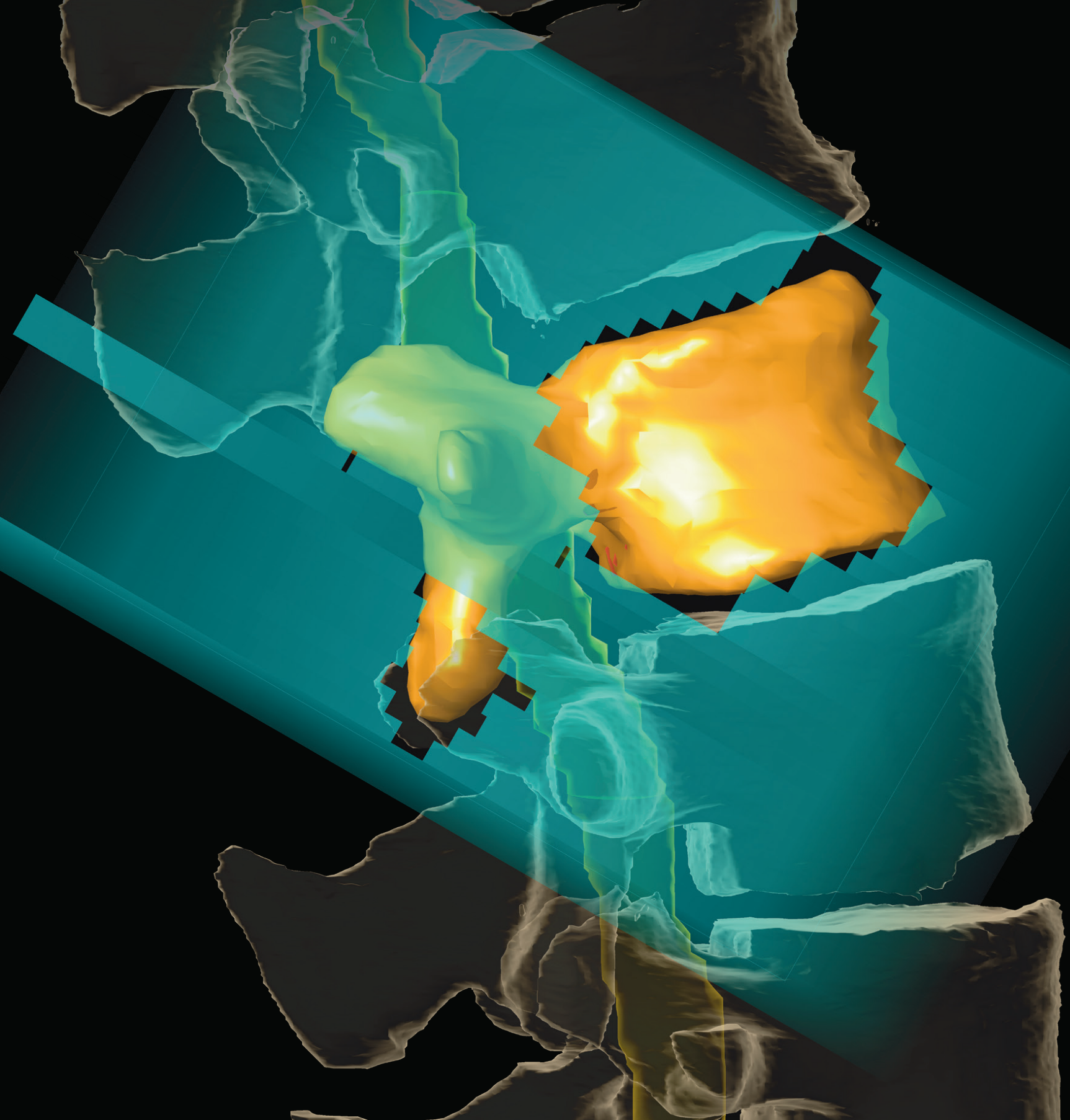

